\title{
Foreign Language and Decision-Making Biases: A Brief Exploration of the Relationship between Emotions, using a Foreign Language, and Decision-Making
}

\author{
Mohamed Hussein ${ }^{\mathrm{a}}$
}

Contrary to what traditional normative decision-making models claim, a change in how a given problem is framed influences the outcome of the decision-making process. Neurological research has shown that during this so-called framing effect, brain structures that are typically associated with the emotional system are activated, suggesting an emotional dimension to the processes that underlie heuristic biases. Recent studies have also shown that thinking in a foreign language can reduce physiological responses typically associated with emotional reactions. This research paper explores the relationship between using a foreign language, emotions, and decision-making in order to determine whether using a foreign language can reduce decision-making biases. More specifically, this paper reasons that using a foreign language can reduce some heuristic biases provided that the given problem is emotionally-laden.

Keywords: Foreign Language; Decision-Making; Heuristics

Emotions are typically neglected by traditional decisionmaking theories that assume the rationality and invariance of decision makers. The expected utility theory, for example, maintains that when decision makers are faced with various options, decision makers tend to choose the option that maximizes their expected utility (Colman, 2009). Tversky and Kahneman's (1981) Asian Disease problem demonstrates an instance wherein decision makers divagate from the rationality claimed by the expected utility theory. In their experiment, participants were divided into two groups where they were told of the outbreak of a hypothetical Asian disease that would kill 600 people and were asked to choose one of two options. In the gain frame group $(\mathrm{G} 1=152)$, participants chose between option A, which would save 200 lives, and option B, which had a $\frac{1}{3}$ probability of saving 600 lives and $\frac{2}{3}$ chance of saving no one. In the loss frame group ( $\mathrm{G} 2=155)$, participants were given the options of four hundred people dying (Option $\mathrm{C}$ ) or a $\frac{1}{3}$ probability that no one would die and a $\frac{2}{3}$ probability that all 600 would die (Option D). According to the expected utility theory, there should be no statistically significant difference between the frequency of choosing option $\mathrm{A}$ and $\mathrm{C}$, since they have the same numerical value (in C, 400 dead out of 600 people $=200$ saved $=\mathrm{A}$ ). Subsequently, we would expect no discrepancy in the frequency of choosing $\mathrm{B}$ and $\mathrm{D}$, since the expected value of lives saved is the same $\left(\frac{1}{3} \times 600=200\right.$ alive in $B=$ $\frac{2}{3} \times 600=400$ dead in $D$ ). However, the results showed that $72 \%$ of participants in G1 chose option A, while only $22 \%$ of participants in $\mathrm{G} 2$ chose option $\mathrm{C}$. This framing effect suggests that decision makers are risk-averse when a given problem is framed in a gain frame and risk-seeking when the same problem is phrased in a loss frame. Clearly, this undermines decision-making theories that assume participants' consistent rationality and suggests that an emotional system may be involved in decision-making. De Martino, Kumaran, Seymour, and Dolan (2006) verified the behavioral results obtained by Tversky and Kaheman through an altered gambling version of the Asian disease dilemma. In this version, each of the twenty subjects, inside an fMRI scanning machine, was shown a series of images and asked to make a choice between two options. The first image was a blank black image, the second was a black image with the text "YOU RECEIVE \$50," and the third differed based on the frame; in the gain frame, it was a symmetrically divided image between "KEEP \$20" (option A) and a pie chart that visually represents a $\frac{1}{3}$ probability of keeping all $\$ 50$ and a $\frac{2}{3}$ probability of losing all, with the word "GAMBLE" over the whole pie (option B). In the loss frame, one half read "LOSE $\$ 30$ " and the other had the aforementioned pie chart. Subjects, as anticipated, were risk-averse in the gain frame and riskseeking in the loss frame. More importantly, this study found a statistically significant correlation between the activation of the amygdala and subject's tendency to exhibit the framing effect, providing neurobiological bases for believing that decisions affected by the framing effect are driven, at least partially, by an emotional system.

In a seemingly unrelated line of research, C.L. Harris (2004) investigated the emotionality of words in bilingual speakers' first language and second language through measuring the bilinguals' skin conductance response (SCR). $\mathrm{SCR}$ is the increase in conductivity of the skin as a part of the physiological response activated by the autonomic nervous system in the presence of external stimuli that may be dangerous. Curiously, Harris found a statistically significant increase in SCR when subjects heard or read taboo words and reprimands in their native language than when they heard equivalent words in their second language. This suggests that a more emotional reaction is produced when the stimulus is in the subject's dominant language. Combined with De Martino's et al. (2006) findings, this study has implications for using a foreign language during decision-making processes. Based on these results, one would predict that making a decision that involves affective heuristics in a foreign language would alter one's decisions. There are two 
plausible outcomes: firstly, since thinking in a second language increases the cognitive load on the brain, it may be the case that the brain will rely more on heuristics in order to economize on its use of cognitive energy, increasing the decision-making bias. Alternatively, since visual and audio stimuli in a second language were found to reduce the emotional attachment of subjects (Harris 2004), one might expect there to be less bias as the affective heuristic component of the decision-making process would be reduced.

Keysar, Hayakawa, and An (2012) explored the possibility of a foreign language influencing heuristics biases through replicating the Asian disease problem with bilinguals who spoke the same native language and the same foreign language. Participants were divided into two groups: the controlled group received the problem in the native language, while the experimental group received the problem in the foreign language. There was a statistically significant framing effect in the controlled group, wherein $77 \%$ of gain frame participants preferred option A as opposed to only $47 \%$ of the participants in the loss frame choosing option C. However, when participants received the Asian disease problem in a foreign language, the framing-effect asymmetry was no longer present; this indicates that making a decision in a foreign language reduces heuristic biases resulting from the framing effect.

One valid concern concerning these results is that participants in the controlled group might have simply chosen their answers randomly rather than exert additional effort in reading, understanding, and deciding in a foreign language. The authors had an additional experiment that contravenes the possibility of participants choosing at random: an additional gain-framed question was included in the foreign language in which participants were to choose between two alternatives, the latter of which clearly yielded a higher expected value. If participants' choices were random, then the data would reflect no clear preference for option B. However, only $14 \%$ of participants chose option $\mathrm{A}$ over option $\mathrm{B}$, reinforcing the validity of the conclusion that using a foreign language diminishes the framing-effect.

These results were successfully replicated by Costa, Foucart, Arnon, Aparici, and Apesteguia (2013). Moreover, the authors extended their inquiry into whether using a foreign language might influence psychological accounting, the way individuals categorize economic outcomes and its subsequent effects on the economic decision taken. Such an effect was tested by means of two problems: the discount problem and the ticket problem. In both problems, half of the 280 participants received the scenarios in their native language and the other half in their foreign language. The discount problem, participants were asked to imagine that they were buying a jacket for 125 euros and a calculator for 15 euros. They then found out that the calculator was being sold for only 10 euros at a nearby shop. The question was whether they would make the trip or not. The second scenario of the discount problem was quite similar, except that the 5 euros discount was on the 125 euros item not the 15 euros item.

Rationally, the discount is the same: 5 euros off a purchase of a total of 140 euros and hence, a perfectly rational decision-maker would either make both trips or neither. However, it is often the case that individuals calculate the discount minimally - out of the percentage of the item that has the discount-rather than globally. In other words, individuals choose to go to the other shop to save $\$ 5$ out of $\$ 15(33.3 \%)$ rather than to save $\$ 5$ of $\$ 125$ (4\%) because $33.3 \%$ is psychologically accounted for as substantially more than a mere $4 \%$. A clear framing effect is reflected in the $28 \%$ difference between the percentage of individuals who indicated that they would go to the other store in the first scenario but not in the second scenario. Such a discrepancy vanished in the other half of the 282 participants who took the test in their foreign language. This shows that thinking in a foreign language diminishes the tendency to perform biasedly in a psychological accounting problem and further suggests that, indeed, using a foreign language reduces some decisionmaking biases.

Interestingly, such results were absent from the second problem that tested for the effect of using a foreign language on psychological accounting. In the ticket problem, 280 participants were asked about the likelihood of two scenarios: The first was about a woman who has brought two tickets to go to the theatre, 80 euros each. Upon her arrival at the theatre, she discovers that she has lost her tickets. Participants were then asked whether they predict that the woman would buy two more tickets. In the second scenario, wherein the woman loses the 160 euros with which she was going to buy the tickets, participants were asked if they thought she would buy the tickets using her credit card. Generally, individuals believe that the woman is more likely to buy another two tickets when she had lost the money more than the other scenario. This can be attributed to subjects' tendency to account for the price of the lost tickets as part of a total psychological price of tickets, whereas the lost money, which could have been spent on other objects, is not included in the total psychological price of the tickets. In other words, people estimate the cost in the first scenario as $\$ 160$ and as $\$ 320$ in the second scenario, thus viewing the former as more likely to occur despite the same economic cost of $\$ 320$ for the woman in both scenarios. This bias was reflected in a clear framing effect seen in the native language group. In the foreign language group, a framing effect was also observed, although to a lesser extent.

The appearance of a framing effect (although less severe) in the foreign language group for the ticket problem is surprising considering the disappearance of such effect in all the aforementioned experiments. Explaining the exact reason behind these results is beyond the scope of this paper. Nonetheless, a possible explanation might be related to the use of language, more specifically pronouns, in the ticket problem. The main discrepancy between the ticket problem and others is that the subject of the dilemma was not the participant themselves but rather a stranger woman, a third person. Studies indicate that the use of the second person pronoun you increases spatial awareness and internalizes emotions of the reader (Brunye 2011). Thus, perhaps the absence of the foreign language effect can be attributed to a change in the level of emotionality of the problem due to its phrasing. At a cursory glance, this discrepancy would be expected to have an opposite effect to what is observed. That is, if the foreign language effect is due to a decrease in the reliance on heuristics that are rooted in an emotional system, providing further emotional distance should yield a weak framing effect in the native language group and no framing effect in the foreign language group. It could be the case that 
the foreign language effect takes place only after a certain threshold of emotional intensity. In other words, perhaps due to the phrasing of the ticket problem, the emotionality of the problem was so low than there was no significant difference between answering the question using a native language or a foreign language. This interpretation seems consistent with results obtained from bilinguals' performance on the cognitive reflection test, which consists of impersonal questions that assess the subject's ability to suppress an incorrect intuitive answer to generate a correct logical answer. The number of incorrect and correct answers was fairly similar in the native and the foreign language groups for this unemotional test (Costa et al 2014). In short, if the foreign language effect relies on emotions and the problem is inherently low on emotions, using a foreign language probably will not decrease heuristic biases.

The framing effect, among other heuristic biases, was diminished when subjects took decision-making tests in their foreign language. A probable explanation of this phenomenon is that using a foreign language incites a less emotional response and consequently decreases the interference of the emotional system with the decision-making process. The exceptions wherein no foreign language effect was detected can be attributed to an inherent lack of emotionality in the problem that renders using a foreign language superfluous in changing the outcome of the decision-making process. This hypothesis is far from conclusive and calls for further investigation to verify whether, indeed, there is an 'emotional threshold' after which the foreign language effect can be observed.

\section{References:}

Brunye, T. T. (2011-08-01). Better you than I: Perspectives and emotion simulation during narrative comprehension. Journal of cognitive psychology (Hove, England), 23(5), 659-666.

Colman, A. M. (2009). A dictionary of psychology. Oxford University Press.

Costa, A. (2014-02-01). "Piensa" twice: On the foreign language effect in decision making. Cognition, 130(2), 236254.

Damasio, A. R. (2005). Descartes' error: Emotion, reason, and the human brain. London: Penguin.

Harris, C. L. (2004). Bilingual speakers in the lab: Psychophysiological measures of emotional reactivity. Journal of Multilingual and Multicultural Development, 25(2-3), 223-247.

Keysar, B. (2012-06-01). The Foreign-Language Effect: Thinking in a Foreign Tongue Reduces Decision Biases. Psychological Science, 23(6), 661.

Tversky, A., \& Kahneman, D. (1981). The framing of decisions and the psychology of choice. Science, 211(4481), 453-458. 